\title{
Exploring Enforced Collaborative Agreement in Gaming with Young People
}

\author{
Daniel Fitton \\ Chigbo Onyinyechukwu \\ University of Central Lancashire \\ Preston, UK \\ dbfitton@uclan.ac.uk \\ ONChigbo@uclan.ac.uk
}

\begin{abstract}
This paper explores the concept of Enforced Collaborative Agreement (ECA) whereby players in a digital game must synchronously 'agree' on their controller inputs in order to interact. The focus of this paper is on the collaborative strategies young people (aged 14-16 years) adopted to reach decisions and control during gameplay. A two player collocated game supporting three different interaction methods has been studied. Video analysis of gameplay, along with post-gameplay interviews, surveys and gameplay interaction logs were used to gain insights into player behavior. The key contributions of the paper are an understanding of six key strategies players adopted to reach agreement within an ECA game, a set of more general issues related to the ECA gameplay, and an exploration of the impact of different interaction methods on gameplay experience. The work highlights the potential benefits of ECA in alleviating the often solitary nature of children's computer use.
\end{abstract}

Collaboration, Enforced Collaborative Agreement, Interaction Design, Co-location, Children.

\section{INTRODUCTION}

Since the early 1980s educators have sought to leverage the motivation and engagement of computer games to engage children with learning $[27,28]$. Since then the potential benefits of using computer games in the classroom have been shown to be increased children's interest in learning [26], facilitation of individualized learning [18], along with enhanced engagement [8], and there is great interest in the field of Serious Games which deals with the design of digital games used for purposes other than entertainment [25]. However, one major concern is that most computer games are solitary which can impair social connections for children and may lead to reduced social skills [7]. Historically this problem has been compounded by the single-user nature of the personal computers and the isolated location of computers [28].

While solitary gameplay can be very beneficial, as highlighted above, it would be most preferable to encourage children to communicate and collaborate with one another and develop positive social skills. Collaboration with others forms a large and important part of our lives from childhood games, through education, to workplaces and beyond. Collaboration is known to have benefits, for example, within educational contexts it is shown to enhance problem solving skills and motivation in children, encourages development of skills of critical thinking, communication, coordination and conscious knowledge construction mechanisms [7]. Although these benefits can be compromised if the contributions of some group members dominate while others are marginalized [2].

In this paper, the concept of Enforced Collaborative Agreement (ECA) is introduced and explored. ECA is a type of interaction where synchronous agreement between the input devices of all players is required in order to interact with a digital game. For example, within an ECA game all players must agree to press 'left' button at the same time in order to move the character left. The context of this work is within co-located settings where face-to-face negotiation, outside of the game environment, is required to reach agreement. The authors envisage ECA as having potential to foster new kinds of faceto- face multiuser collaborations around interactive 
applications that traditionally only support a single user/player. Using an approach such as ECA solitary single- player electronic games can be made collaborative and social thus offering a rich way for players, particularly children, to improve their language and communication skills. The work reported here has also shown that ECA has the potential to encourage equitable participation. These benefits may be particularly valuable for users in the context of educational environments and serious games. While existing work has studied collaboration in enforced situations [15], [19], ECA is novel in that players have to synchronously agree with their control inputs in order to interact. Additionally, in our work we compare three different types of controller (a traditional gamepad, a tangible device, and a dance mat controller). The primary aim of the research reported in this paper was to understand the strategies young people adopted to reach decisions on control within ECA games, in order to begin to explore this new design space. The secondary aim of this work was to study game play experience in an ECA game.

\section{RELATED WORK}

The term 'collaboration' can be used to describe a wide range of behaviors and generally refers to any activity performed together by a pair or a group of individuals. Lipponen [16] provided two approaches adopted by researchers towards the definition of collaboration: collaboration as a process of participation in collective activities and collaboration as a special form of interaction. In this work, collaboration is viewed as a special kind of interaction stressing the idea of mutual engagement of those involved. Roschelle and Teasley [23] clearly differentiated collaboration from cooperation stressing that cooperation requires division of labor where each person is responsible for a portion of a problem while collaboration emphasizes on a mutual engagement of participants in a coordinated effort to solve a problem together. Furthermore, Kerawalla et al. [14] highlighted that when a task is collaborative participants make joint decisions. However, in cooperative task situations participants divide the task into parts, taking responsibility for their own part, and may come together to fit these parts together.

Researchers have highlighted collaboration as an important skill for children's learning and development (e.g. [5],[22]). A large body of previous work has explored collaboration with children in colocated settings. While some of this concentrated on the design of interfaces to support co- present collaboration (e.g. [10][1]) the majority focused on the use of technology to support children's collaborative interactions (e.g. [12],[14],[11]). These studies identified different collaborative behaviors exhibited by children when they interact in varying collaborative settings. For example, Jamil et al. [12] observed a group of children whilst they designed a seating plan for their classroom using single touch and multi-touch tabletops, they identified taskfocused and turn-taking discussions in the multitouch and single touch conditions respectively. Inkpen et al. [11] found that when provided with multiple mice children appeared to participate more actively and exhibited high levels of engagement. However, providing participants with multiple mice without enforcing collaboration does not guarantee highly collaborative behaviors. Dual representations of a task were explored in [14], although children used multiple mice when interacting with a single task representation it was observed that they exhibited behaviors that were not conducive to joint understanding such as parallel working and more domineering behaviors with minimal discussions than with the separate control of shared spaces (SCOSS).

Zagal et al. [28] classified games into three categories: competitive, cooperative and collaborative games. In competitive games, players form strategies that directly oppose those of the other players in the game. Cooperative games provide opportunities for players to achieve a winwin situation by working together whereas in collaborative games, all players work together and wins/loses as a team. While most games are primarily for entertainment purposes, serious games have more than just the story, art and software but incudes activities that educate or instruct, thereby imparting knowledge and skill [29] and have been successfully applied in several areas including the military, government, educational, corporate and healthcare [25]. Within gaming contexts, collaborative mechanics have become prominent with the likes of massively multiplayer online games (MMOGs) such as World of Warcraft, Lord of the rings, Minecraft etc. However, most of these games encourage rather that enforce collaboration [28]. Pianesi et al. explored the design and evaluation of a collaborative Puzzle game to foster collaboration and social skills in children with ASD [19]. Their work 
employed an enforced collaborative mechanism, and focused on children with Autism Spectrum Disorder (ASD). More recently, Margel and his colleagues employed the concept of ECA in an online experiment, Twitch Plays Pokemon, where millions of players simultaneously controlled one character in a game [17]. These are the closest works to that presented in this paper but differs in so far as in this work we explored enforced collaboration in co-located settings using a range of data gathering approaches (i.e. video analysis of gameplay, and use of graphs of gameplay data as prompts during unstructured interview sessions with children), a range of interaction techniques, and a focus on agreement between pairs of children with no diagnosed developmental difficulties collaborating during game play. While all papers previously cited have used a wide range of approaches and formed a valuable set of findings to inform this work, no study has yet explored synchronous forced collaboration with children in the way that is described here.

\section{ECA GAME DESIGN}

To enable exploration of ECA a simple game (based upon 'Space Invaders') was created that could easily be integrated with a range of interaction possibilities. The Space Invader game was chosen as a basis because of its simplicity. It was anticipated that such a simple game would take little time for participants to learn (with very minimal control inputs) and allow for focus on the collaborative aspects of the gameplay whilst offering some level of engagement. Additionally, the game can be controlled with 3 inputs (left, right, fire), which simplified gameplay and agreement on controller input. Adobe Flash was used to rapidly prototype a game with both collaborative and single-player modes. In the collaborative version all players must synchronously agree in their controller inputs in order to control the 'cannon' in the game e.g. both players must be pressing the fire button at the same time in order for the cannon to fire. For simplicity the game had just one level. During development the game was piloted twice, in the first pilot 15 children aged 7-8 years played the single-player version of the game individually using a keyboard. In a second trial 42 children aged 11-15 grouped in pairs played the game in the collaborative mode using game pads. In both pilots each period of gameplay lasted 2 minutes, the expected gameplay time to be used in later studies. A short questionnaire was used at the end of each gameplay period to elicit feedback on several aspects of the gameplay.

In order to assist participants in synchronously agreeing on controller inputs the concept of an 'interaction map' was conceived by the authors (top right of Figure 1). The interaction map consists of three objects; two triangles pointing to the left and right side of the screen (representing the left and right direction respectively) and a large circle (representing the 'fire' command) in between them. Dots appeared in these areas when the players pressed the corresponding buttons on their controllers, a color code for the dots was used to distinguish between players. The intention was to enable participants to see their own control inputs alongside those of their co-player during gameplay.

A different style of interaction map was required for the tangible input methods as it relied on a tilting movement rather than button presses. The visualization, shown in Figure 2, not only made the actions of other players visible but also assisted users in understanding the degree of physical tilt of the controller required to generate the left/right movement inputs.

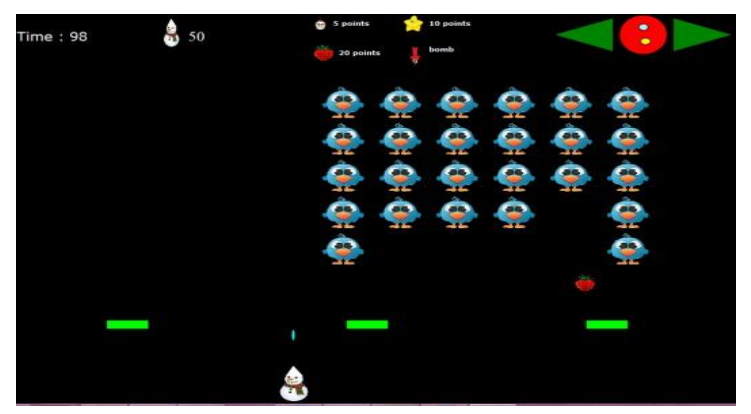

Figure1. 'Space Invaders' Game

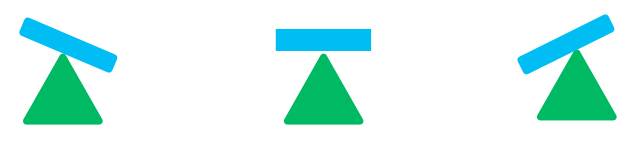

Figure2. Visualization for Tilt Input

\subsection{Controller Design}

Three different interaction techniques were chosen to represent a range of different interaction possibilities: traditional (a PC-based game pad), tangible (an object incorporating a Nintendo Wiimote for motion sensing) and embodied (a dance mat game controller). The Wiimote was used in order to sense movement wirelessly with ease and was hidden to prevent children from realizing that it was 
a standard game controller that they were likely to be familiar with. The embodied interaction method was a dance mat that supported body based interaction (and which has not been widely used in studies with children). Other practical constraints such as cost and ease of integration also influenced the choice of the technologies. The controllers had color coding corresponding to those of the interaction map.

\section{STUDY 1}

Twelve young people aged between 14 and 16 years old participated in the study. The participants were grouped in pairs resulting in two boy-boy groups, two girl-girl groups and two mixed groups. To maintain the anonymity of participating children, group codes were used resulting to groups A-F.

The study was one of a set of activities carried out in a MESS day [9], where a whole school class visited a lab and moved between different activities. It began with each participant completing a background questionnaire on their experience related to technologies and gaming. The participants were then asked to stand in a marked area two meters away from a screen onto which the game was projected. This ensured that the participant's positions from the screen remained consistent across groups. The researcher then explained the rules of the game and that the game would be played using three different controllers. For the gamepad, left and right movement was controlled using the d-pad buttons with one of the colored buttons used to fire. For the tangible device the controller was tilted to the left or right respectively and firing was achieved by pressing a physical button on the device. The dance mat used the left/right direction pads for movement and the up direction pad for fire. A within-subject design approach in which pairs played both the single and collaborative versions of the game using all three controllers (game pad, dance mat and tangible) was used. In order to counterbalance for learning effect, a $3 \times 3$ Latin square design approach was used to select the order in which each pair played [4]. This resulted in one-third of the participants playing the game using game pad first followed by tangible and dance mat; one-third of the participants playing the game using dance mat first followed by game pad and tangible; one-third of the participant playing the game using tangible first followed by dance mat and game pad. For training purposes, each participant in a pair played the single player version individually for 30 seconds before playing the collaborative versions for two minutes each. Each pair completed an evaluation form (one per participant) at the end of each session to capture their thoughts on the technology using Smileyometer and Again-Again table [20]. The evaluation form also contained questions related to collaboration, familiarity with partner, awareness of the interaction map that was included on the screen, preference for and enjoyment of the game. Afterwards, each pair completed a Funsorter based on which controller they 'liked the most', 'was most fun' and 'was easiest to play with' [20]. The participants were observed all through the entire sessions. Furthermore, an attempt was made to engage the participants in informal discussion about how they reached agreement at the end of each session.

\subsection{Analysis}

The observational data collected during the study was analyzed with another researcher experienced in coding qualitative data to reduce bias. The researchers adopted a thematic analysis method where the observational data were unanimously coded and categorized into themes [3]. The themes obtained were taken to a third party (senior researcher) for validation. The frequency of use (FUS) scale [13] was used in the background questionnaire to measure how often participants played computer games on various platforms. Answers were coded as 4 for 'everyday', 3 for 'a few times a week', 2 for 'once a week', and 1 for 'never'. The Smileyometers were coded in an ordinal way on a 5-point scale, where $5=$ 'brilliant', $4=$ 'really good', $3=$ 'good', 2 = 'not very good and ' 1 = 'awful'. The Again- Again results were coded as 3 for 'yes', 2 for 'maybe' and 1 for 'no'. The Funsorters completed by the participants were coded as 3 for the highest ranked, 2 for the next and 1 for the lowest for each of the construct.

\subsection{Results}

In this work we use $X_{t a n}, X_{g p}$ and $X_{d m}$ to represent groups playing the game with the three different controllers. $X$ ranges from $A-F$ and represents each of the 6 groups that participated in the study. Also, we used $\mathrm{C}_{\mathrm{i}}$ to represent the participants where i ranges from $1-12$.

Before the start of the game only a single group attempted to strategize, with one of the participants 
in $D_{\tan }$ observed instructing his partner on how they would play the game: "I count one and you tilt left, two right, three shoot." During game play in groups $B_{t a n}, D_{t a n}, E_{t a n}, F_{t a n}, A_{g p}, B_{g p}$ and $D_{g p}$ one participant was observed directing the other participant (who then obeyed), for example "Left, right, left, left shoot..." We term this 'dominating behavior' as one of the participants controlled the interaction through verbal instructions while the other passively obeyed. In contrast, participants in $\mathrm{C}_{g p}$ were observed to begin playing silently without engaging in any discussion but a strategy was soon suggested by one of the participants "Ok, maybe I shout and you press shoot." Participants in $A_{t a n}, A_{d m}, B_{d m}, C_{d m}, D_{d m}$, $C_{g p}, D_{g p}$, and $F_{g p}$ were all observed to glance intermittently at each other's controllers before looking at the screen. One of the participants in $E_{g p}$ reported noticing the interaction map on the screen however it cannot be confirmed here if they had used the map to inform decision on what actions to take. One of the participants in $\mathrm{F}_{\mathrm{gp}}$ used a 'telling by showing' strategy, showing his partner what to do using his controller while the partner watched. Participants in $A_{d m}$ and $D_{g p}$ asked for help from the researcher while one child in $B_{g p}$ was observed pointing at the screen on two occasions during play.

$83 \%$ of the participants indicated that they noticed the interaction map while the remaining $17 \%$ did not. Also, $70 \%$ of those that noticed the map indicated they knew the purpose of the map, while $30 \%$ did not. However, it cannot be concluded that the participants who noticed the map used it to reach decisions and control during game play. Responses to the post-gameplay evaluations showed that $50 \%$ rated dance mat as 'brilliant' but of those $33 \%$ would not like to play the game again with the dance mat (the remaining $67 \%$ were indecisive). Figure 4 shows for each construct ('liked the most', 'was most fun' and 'was easiest to play with'), how many participants ranked each controller highest. Similar to the results obtained using the Smileyometer, the dance mat appeared to be the most fun controller: with $67 \%$ of the participants ranking the dance mat highest on the 'most fun' construct. Also, the game pad seemed to be the controller that was easiest to play the game: with $58 \%$ ranking it highest on the 'easiest to play' construct. It cannot be concluded in this study which controller was preferred as $42 \%$ ranked both the tangible and game pad highest on the 'like the most' construct.

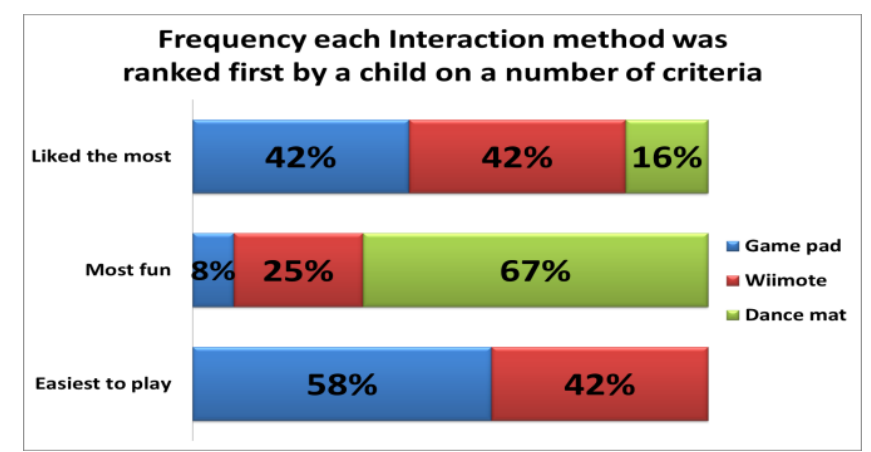

Figure 4. Rankings of the three controllers

The initial study served as a platform to test the methodologies for measuring ECA as well as monitoring the operation of study design. Results showed that the participants did not have any major issues across the three controllers. However, it was discovered that the methods used to measure collaboration were limited in terms of insights into participants collaborative behaviors.

\section{STUDY 2}

Eight participants aged 15 years (six boys and two girls) participated in the study. The participants were selected and grouped in pairs by the class teacher resulting in groups of three boy-boy groups and one girl-girl group. Group and participant codes were used for the sake of anonymity.

As with the earlier pilot study, a within-subject design approach in which pairs played both the single and collaborative versions of the game using the three controllers (game pad, dance mat and tangible) was used. The study began with each pair completing a background questionnaire (same as the one used in the initial study). The single player training condition and the collaborative conditions of the game were increased to one and four minutes respectively. Each pair completed a post-test evaluation form and Funsorter same as was used in the initial study. The participants were then interviewed using the graphs generated from the log file data obtained during game play (showing interactions and periods of agreement) as prompts.

\subsection{Analysis}

The video data was analyzed using a whole-to-part inductive approach [6] whereby the video data was scrutinized to identify the strategies participants adopted to reach decision and control. The annotation scheme used consisted of 75 parent tiers. The parent tiers included orthographic transcript of participants conversations (verbal 
annotation), hand gestures, eye-gaze of each child while playing with each controller (non-verbal annotation) and the leg movements of each participant while playing with the dance mat. Verbal and non-verbal annotations were distinguished according to controller type as it was envisaged that different strategies would be adopted for each controller. To gain further insights 'Collaborative Networks' were adopted. Collaborative Networks were specifically developed to address some of the deficiencies of existing methods for analyzing and presenting complex collaborative processes consist of both participants talk and actions visually represented in terms of the evolutionary path interactions have taken [24]. A coding scheme grounded in the video data was developed over a series of iterations as shown in Table 1.

The descriptive data (speech and action) alongside their accompanying codes were represented within the collaborative networks to give a clear picture of the behaviors participants exhibited during interaction with the game. We used these approaches to investigate whether the collaborative behaviors in video data reinforce those found in the initial study. The participants responses during the interview sessions were transcribed verbatim and analyzed using thematic analysis [3]. The researchers adopted an inductive approach during the analysis process where the participants responses were unanimously coded and categorized into themes. Analysis of the data obtained through Smileyometer, Again-Again, funsorter and the questionnaires followed same approach as in the initial study.

\subsection{Results}

We use the same notations as earlier to represent groups that played the game with the three different controllers. However, $X$ ranges from $A-D$ and $i$ from 1-8. We present segments of the collaborative networks in tables to illustrate extracts of participants' speech and action. The first three (left side) columns are associated with the player on the left of the screen during gameplay, while the second three (right side) columns are associated with the player on the right of the screen during gameplay. The first and last columns show the time codes associated with each row. The second and fifth columns show the speech and actions from participants. The third and fourth columns indicate the code assigned to the situation (form Table 1) and the arrow shows the direction in which participants is giving their attention, typically to their partner but sometimes to the screen (shown by an arrow pointing downwards).

\begin{tabular}{|c|c|c|}
\hline Verbal Instruction & Code & Explanations \\
\hline $\begin{array}{l}\text { Giving } \\
\text { direction/instruction }\end{array}$ & Gi & Partner directs or instructs \\
\hline Suggestion & Su & Partner makes a suggestion \\
\hline Disagreement & $\mathrm{D}$ & $\begin{array}{l}\text { Partner gives counter instruction, rejects } \\
\text { suggestions or makes negative comments }\end{array}$ \\
\hline Agreement & $\mathrm{Ag}$ & $\begin{array}{l}\text { Partner affirms to instructions given, } \\
\text { accepts suggestions or makes positive } \\
\text { comments }\end{array}$ \\
\hline Explanation & Ex & Explaining own or partner's action/intent \\
\hline $\begin{array}{l}\text { Peripheral } \\
\text { Verbalization }\end{array}$ & $\mathrm{Pe}$ & Verbalizations not related to collaboration \\
\hline Not Talking & NT & $\begin{array}{l}\text { Playing silently, no communication via } \\
\text { talking }\end{array}$ \\
\hline Noticed Map & $\mathrm{Nm}$ & $\begin{array}{l}\text { Verbalizations related to the map on the } \\
\text { screen or those accompanying pointing at } \\
\text { the map on the screen }\end{array}$ \\
\hline Enquiry & $\mathrm{E}$ & 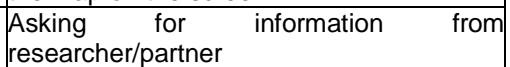 \\
\hline Response & Res & Response to partner's enquiry \\
\hline
\end{tabular}

Table1. Coding Scheme

Prior to start of game play, it was observed that participants in $\mathrm{B}_{\tan }$ engaged in negotiations in order to adopt strategies for game play as seen in their excerpt in Table 2. participants 3 suggested to participants 4 the direction they should start from. Initially, participants 4 did not accept the suggestion but clearly said what direction he wanted to go. participants 3 disagreed and went further to provide some explanation to his suggestion which made participants 4 to accept the suggestion.

During gameplay, several occasions of conflicts (disagreement) were observed within groups and across controllers. Whilst some of the conflicts were resolved immediately as seen in in $\mathrm{B}_{\mathrm{gp}}$ 's excerpt in Table 3, others took a longer duration and were resolved with explanations as seen in Bdm's excerpt in Table 4. There were several non- conflict situations were a participant affirmed to partner's instructions without further explanations as seen in Agp's excerpt in Table 5. There were indications of dominating behaviors in $A_{g p}, A_{d m}$ and $D_{d m}$ where a participant in a group gave verbal instructions to the partner while the partner passively carried out the instructions. However, this did not continue throughout the rest of the game play session. Also, there were cases where suggestions were made by one participant to another during game play, but these suggestions were not always accepted. In some cases explanations were required to convince the other participant to accept the suggestion as seen in Bgp's excerpt in Table 6. 
Furthermore, it was observed that some participants made enquiries and received responses from their partners and even the researcher regarding game controllers but on one occasion $B_{d m}$ made an enquiry that was specifically about the game play. Only $A_{g p}$ used a telling by showing strategy (where one participants showed his partner what to do using his controller while the partner watched). It was observed that on two different occasions pairs in $\mathrm{C}_{\mathrm{gp}}$, touched each other's controller to make the partner do the same thing. However, each participants responded in a way that did not allow the partner to dominate as seen in Table 7.

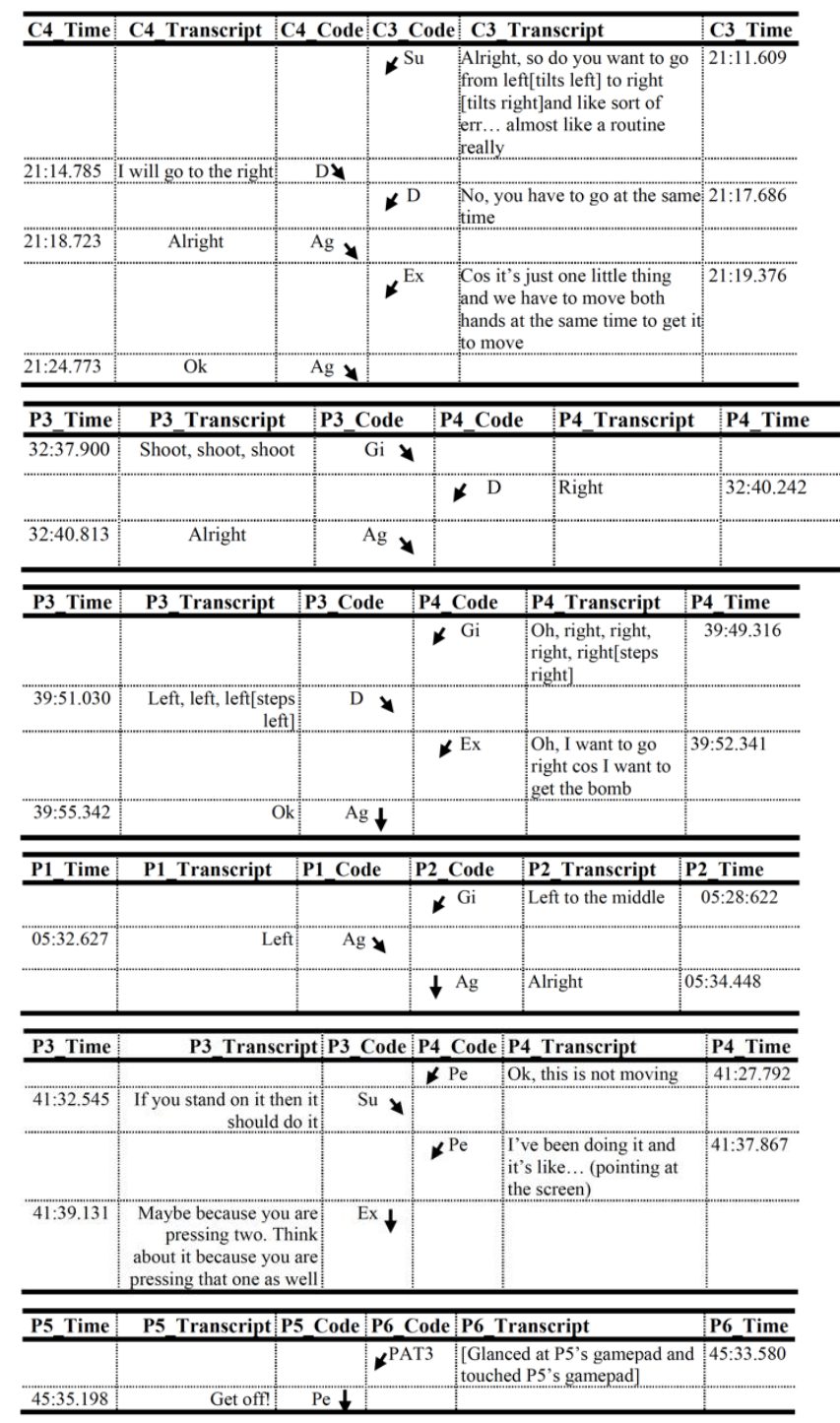

Tables 2,3,4,5,6 and 7. Collaborative Networks

On many occasions, participants gave verbal instructions and pointing instructions (deictic gestures) to their partners during game play. All the participants indicated that they noticed the interaction map on the screen and all of them knew the purpose of the map apart from $C 7$ in group $D$ that did not respond to the question. This was also evident in the

C3: "... if you look at the top right you can see when we are pushing the button at the same time...So I can see when you are pushing left, pushing right."

C4: "You can see it on the top bit [pointing at screen]"

All the behaviors were observed for various groups while playing the ECA game with all the three controllers except for 'Not allowing partner dominate' and 'telling by showing' which occurred only in the gamepad condition in Groups A and C.

\subsection{Experiences}

The participants rating of their fun experience and their responses to the question if they would like to play the game again using the three controllers showed that $63 \%$ of the participants rated the dance mat as 'brilliant' but $40 \%$ out of those who thought the dance mat was brilliant would not like to play the game again with the dance mat (while the remaining $60 \%$ were indecisive). This result is similar to that obtained in the initial study. Figure 5 shows the participants rankings of the three controllers according to the constructs 'liked the most', 'was most fun' and 'was easiest to play with'.

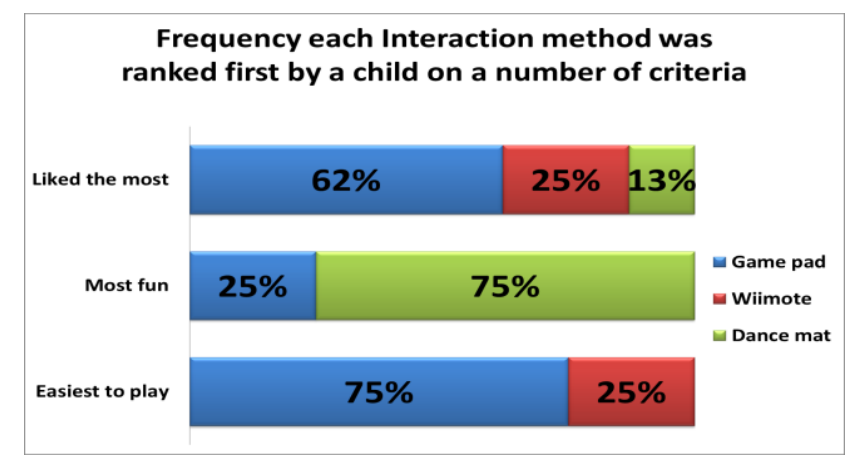

Figure 5. Children's rankings of the three controllers

Similar to the results obtained using the Smileyometer, the dance mat appeared to be the most fun controller as $75 \%$ of participants ranked the dance mat highest on the 'most fun' construct. The game pad appeared to be the easiest to play and the one liked most by the children: $75 \%$ of the participants ranked the game pad highest on the 'easiest to play' constructs and $62 \%$ of the participants ranked the same controller highest on the 'like the most construct'. In addition, those who ranked the dance mat lowest on 'most fun' construct of the Funsorter also rated the dance mat as 'not 
very good' using the Smileyometer. Also, $63 \%$ of those that ranked the dance mat highest on the 'most fun' construct of the Funsorter rated the dance mat as 'brilliant' using the Smileyometer. Further examination of the Funsorter results showed that $88 \%$ of those who ranked a controller highest on the 'easiest to use' construct ranked the same controller highest on the 'liked the most' construct. Two participants ranked the same controller highest on the three constructs.

\subsection{Interview Responses}

Presented in this section are the themes generated from the thematic analysis of responses during the interview sessions with quotes from the raw data indicative of each theme.

1) Strategy: All groups developed strategies to play the game. While some $\left(A_{g p}, A_{t a n}, B_{t a n}, C_{t a n}, D_{d m}\right)$ did this in advance others $\left(B_{g p}, C_{g p}, D_{g p} D_{t a n}, A_{d m}, B_{d m}\right.$, $\mathrm{C}_{\mathrm{dm}}$ ) developed strategies during game play. One of the participants $(C 1)$ in Group A identified that their initial strategy which involved use of the game features influenced how they collaborated using the dance mat. According to $\mathrm{C} 1$ : "... it was just that we decided pressing where they [aliens] wanted to go and then we will say to the other person go right..." In addition, pairs in Group B had no initial strategy however they noticed the map during game play and used it to collaborate as stated by one of the participants in the group "I think we were both trying to do different things and then we looked up at the little bars, the circles which showed us what each other was doing. And then we thought, oh alright I would do what each other was doing."

2) Synchronicity of Response: Synchronicity of response relates to synchronous agreement between players interactions (i.e. pressing the same button at the same time). There seemed to be no serious problems related to this issue in either study. In some cases participants responded quickly to the game but not at the same time as evident in C7s response in Group D while playing with the dance mat: "when there were long spaces, I think we responded really quickly..." and C6 in Group C while playing with the game pad: "we were both pressing it very fast..." One of the participants (C1) in Group A indicated that it took them more time to react when playing with the dance mat as a result of the strategy they adopted i.e. strategized using alien movement, while $\mathrm{C} 6$ in Group C mentioned that it took them: "...a bit to get together..." as they needed to coordinate their interactions while playing with the dance mat. Furthermore, C6 in Group C indicated that it was difficult to coordinate key presses while playing with the game pad: "...I don't think we were pressing the red button at the same time very easily". This issue with the game pad may have arisen due to the typically very short duration of button presses.

3) Accidental Interactions: This refers to when players generate game control inputs not planned or intended. Pairs in Group C and Group A found that they accidentally tilted the tangible controller and that this influenced how they collaborated. This is evident in responses of C5 in Group C "I kept slipping going that way without realizing" $\mathrm{C} 6$ in Group C "Yeah, it's hard to get it really straight" and C1 in Group A: "...sometimes you just accidentally tilt it."

4) Controller Ease of Use: This refers to the players' ability to easily interact with the controllers. The gamepad was reported as the easiest: "I think it's more of a case the buttons are easier to press..., it's easier to go on and off, on and off..." (C4, Group B).

5) Controller Visibility: This refers to how clearly a controller can be seen by the players during interaction. C4 in Group B stated that the size of the tangible controller caused them to easily see what each other was doing as seen in the comment "Yeah, cos it's so big. It's easy to see what the other person is doing. Cos you can see them going like that (gestures)"

6) Familiarity with Controller: This refers to the degree of familiarity participants felt with the controllers. C7 in Group D felt that the game pad had strong single player connotations for them: "it might be that when you've got that kind of controller, your immediate response is to play a single player cos that's how you normally play it at home." Conversely, unfamiliarity was found to foster collaboration, in relation to the tangible and dance mat $\mathrm{C} 7$ in Group D stated "I think with the other two you have to work as a team cos you have never used that kind of equipment before."

\section{DISCUSSION}

The findings from this research revealed a range of different collaborative behaviors exhibited by the participants whilst playing a multiplayer game that supports ECA. The collaborative networks provided a framework for analyzing the video footage from the 
study and developing understanding of the collaborative behaviors [24]. From the collaborative networks six key collaborative behaviors were observed which were identified in study 2 . These included negotiations, verbal suggestions, explanations, enquiries and response to enquiries. These user behaviors are important to consider in the design of an ECA (or similarly collaborative) game in the context of child users. A set of six core themes which influenced participant interactions were identified from post-gameplay interviews in study 2 . These themes were strategy, synchronicity of response, accidental interactions, controller ease of use, controller visibility, and familiarity with controller. The themes span a range of levels and can be used to inform future work in the area of ECA in a range of possible application scenarios.

\section{CONCLUSIONS}

This work explored the concept of ECA (Enforced Collaborative Agreement) whereby all players in a game must synchronously agree in their controller inputs in order to interact with a digital game. ECA has the potential to enable new kinds of collaborative and democratic games and applications, which may be particularly valuable in the areas of educational software and serious games. The main aims of this work were to explore how participants collaborated in order to reach decisions while playing a game with ECA, the different collaborative techniques they used, and gameplay experience in a ECA game. Two main studies were carried out involving a total of 20 participants aged 14-16 years playing an ECA game in pairs, a range of data was collected before, during and after gameplay. Through the use of collaborative networks a set of six collaborative behaviors exhibited by the participants during gameplay were identified, these are of particular value to designers seeking to create collaborative games for young people that require agreement. This work also identified a set of six more holistic issues, which emerged from post-gameplay interviews, that related to the ECA gameplay and are also of value to those working in a similar domain. This work also explored user experience within an ECA game, primarily within the context of three different interaction methods used to play the game (a traditional style game pad, a tangible controller using tilt-based interaction, and a dance mat). Findings showed that participants in Study 1 and 2 found the dance mat the most fun but that the gamepad was the easiest to use. In terms of the collaborative patterns observed, we found no clear differences between the three interaction methods.

It is our hope that academics and developers may take inspiration from our work and utilize the ECA approach to help make games and applications for young people both more collaborative and democratic. In the future we are keen to build upon the insights and understandings of this work in the context of other games, serious games and applications intended for child and teenaged users.

\section{REFERENCES}

1. Antle, A.N., Wise, A.F., Hall, A., et al.Youtopia : A Collaborative, Tangible, Multi-touch, Sustainability Learning Activity. Proceedings of the 12th International Conference on Interaction Design and Children, (2013), 565-568.

2. Bowman, B., Elmqvist, N., and Jankun-Kelly, T.J.Toward Visualization for Games: Theory, Design Space, and Patterns. IEEE transactions on visualization and computer graphics 18, 11 (2012), 1956-1968.

3. Braun, V. and Clarke, V.Using thematic analysis in psychology. Qualitative research in psychology 3, 2 (2006), 77-101.

4. Breakwell, M.G., Hammond, S., and FifeSchaw, C., eds.Research methods in psychology. sage, London, 2000.

5. Daiute, C. and Dalton, B.Collaboration Between Children Learning to Write: Can Novices Be Masters? Cognition and Instruction 10, 4 (1993), 281-333.

6. Erickson, F.Definition and analysis of data from videotape: Some research procedures and their rationales. In Handbook of complementary methods in education research. 2006, 177-205.

7. Ferguson, C.J.Blazing angels or resident evil? Can violent video games be a force for good? Review of General Psychology 14, 2 (2010), 6881.

8. Griffiths, M.The educational benefits of videogames Videogames have great positive potential in. Education and Health 20, 3 (2002), 47-51.

9. Horton, M., Read, J.C., Mazzone, E., Gavin, S., and Fitton, D. School friendly participatory research activities with children. In $\mathrm{CHI}$ '12 
Extended Abstracts on Human Factors in Computing Systems, (2012), 2099-2104.

10. Hourcade, J., Bederson, B., Druin, A., and Taxen, G.KidPad: collaborative storytelling for children. CHI'02 extended abstracts ..., (2002), 500-501.

11. Inkpen, K., Ho-Ching, W., Kuederle, O., Stacey, S., and Garth, S. This is fun! we're all best friends and we're all playing: supporting children's synchronous collaboration. ... for collaborative ..., (1999).

12. Jamil, I., O'Hara, K., Perry, M., Karnik, A., and Subramanian, S.The effects of interaction techniques on talk patterns in collaborative peer learning around interactive tables. Proceedings of the 2011 annual conference on Human factors in computing systems - CHI '11, (2011), 3043-3052.

13. Kano, A., Horton, M., and Read, J.Thumbs-up scale and frequency of use scale for use in self reporting of children's computer experience. Proceedings of the 6th Nordic Conference on Human-Computer: Extending Boundaries, (2010), 699- 702.

14. Kerawalla, L., Pearce, D., Yuill, N., Luckin, R., and Harris, A.I'm keeping those there, are you?" The role of a new user interface paradigmSeparate Control of Shared Space (SCOSS)-in the collaborative decision-making process. Computers \& Education 50, 1 (2008), 193-206.

15. Light, P., Foot, T., Colbourn, C., and Clelland, I.M.Collaborative Interactions at the Microcomputer Keyboard. Educational Psychology 7, 1 (1987), 13-21.

16. Lipponen, L.Exploring foundations for computer- supported collaborative learning. (2002), 72-81.

17. Margel, M.Twitch Plays Pokemon : An Analysis of Social Dynamics in Crowdsourced Games. (2014).

18. Mayo, M.J.Video games: a route to large-scale STEM education? Science (New York, N.Y.) 323, 5910 (2009), 79-82.

19. Pianesi, B.F., Zancanaro, T.M., Venuti, E.P., Gal, B.S.E., and Weiss, P.L.Collaborative Puzzle Game: a tabletop interactive game for fostering collaboration in children with Autism Spectrum Disorders (ASD). Proceedings of the
ACM International Conference on Interactive Tabletops and Surface, (2009), 197-204.

20. Read, J., Macfarlane, S., and Casey, C. Endurability and Expectations :Measuring Children's Fun. (1999).

21. Read, J.C.Validating the Fun Toolkit: an instrument for measuring children's opinions of technology. Cognition, Technology \& Work 10, 2 (2007), 119- 128.

22. Rogoff, B.Developing Understanding of the idea of Comunities of Learners. Mind, Culture and Activity 1, 4 (1994), 209-229.

23. Roschelle, J. and Teasley, S. The construction of shared knowledge in collaborative problem solving. Computer supported collaborative learning, (1995), 70 - 97.

24. Stanton, D. and Neale, H.The effects of multiple mice on children's talk and interaction. Journal of Computer Assisted Learning, January (2003), 229- 238.

25. Susi, T., Johannesson, M., and Backlund, P.Serious Games - An Overview. Elearning 73, 10 (2007), 28.

26. Villalta, M., Gajardo, I., Nussbaum, M., Andreu, J.J., Echeverría, a., and Plass, J.L.Design guidelines for Classroom Multiplayer Presential Games (CMPG). Computers and Education 57, 3 (2011), 2039-2053.

27. Wittenburg, P., Brugman, H., Russel, A., Klassmann, A., and Sloetjes, H.ELAN : a Professional Framework for Multimodality Research. (2006), 1556-1559.

28. Zagal, J.P. Collaborative games: Lessons learned from board games. Simulation \& Gaming 37, 1 (2006), 24-40.

29. Zyda, M.From visual simulation to virtual reality to games. Computer 38, 9 (2005), 25-32. 